\title{
Development and Validation of a Sensitive GC-MS Method for the Determination of Alkylating Agent, 4-Chloro-1-butanol, in Active Pharmaceutical Ingredients
}

\author{
Koki Harigaya, ${ }^{*, a \dagger}$ Hiroyuki Yamada, ${ }^{a}$ Koji Yaku, ${ }^{a}$ Hiroyuki Nishi, ${ }^{b}$ and Jun Haginaka ${ }^{c}$ \\ a Analytical Research Laboratories, CMC Division, Mitsubishi Tanabe Pharma Corporation; 3-16-89 Kashima, \\ Yodogawa-ku, Osaka 532-8505, Japan: ${ }^{b}$ Department of Pharmaceutical Chemistry, Faculty of Pharmacy, Yasuda \\ Women's University; 6-13-1 Yasuhigashi, Asaminami-ku, Hiroshima 731-0153, Japan: and ' School of Pharmacy and \\ Pharmaceutical Sciences, Mukogawa Women's University; 11-68 Koshien Kyuban-cho, Nishinomiya 663-8179, Japan. \\ Received November 24, 2013; accepted January 3, 2014
}

The analysis of genotoxic impurities (GTIs) in active pharmaceutical ingredients (APIs) is a challenging task. The target detection limit (DL) in an API is typically around $1 \mathrm{ppm}(1 \mu \mathrm{g} / \mathrm{g} \mathrm{API})$. Therefore, a sensitive and selective analytical method is required for their analysis. 4-Chloro-1-butanol, an alkylating agent, is one of the GTIs. It is generated when tetrahydrofuran and hydrochloric acid are used during the synthesis of the APIs. In this study, a sensitive and robust gas chromatography-mass spectrometry (GC-MS) method was developed and validated for the identification of 4-chloro-1-butanol in APIs. In the GC-MS method, 3-chloro1-butanol was employed as an internal standard to ensure accuracy and precision. Linearity was observed over the range 0.08 to $40 \mathrm{ppm}\left(\mu \mathrm{g} / \mathrm{g}\right.$ API), with a $R^{2}$ value of 0.9999 . The DL and quantitation limit (QL) obtained were $0.05 \mathrm{ppm}$ and $0.08 \mathrm{ppm}(0.13 \mathrm{ng} / \mathrm{mL}$ and $0.20 \mathrm{ng} / \mathrm{mL}$ as the 4-chloro-1-butanol concentration), respectively. These DL and QL values are well over the threshold specified in the guidelines. The accuracy (recovery) of detection ranged from 90.5 to $108.7 \%$ between $0.4 \mathrm{ppm}$ and $20 \mathrm{ppm}$ of 4 -chloro-1-butanol. The relative standard deviation in the repeatability of the spiked recovery test was $6.0 \%$. These results indicate the validity of the GC-MS method developed in this study. The GC-MS method was applied for the determination of 4-chloro-1-butanol in the API (Compound A), which is under clinical trials. No 4-chloro-1-butanol was found in Compound A (below QL, 0.08 ppm).

Key words 4-chloro-1-butanol; GC-MS; genotoxic impurity; active pharmaceutical ingredient; trace analysis

The analysis of genotoxic impurities (GTIs) in trace amounts is of increasing interest to pharmaceutical industries owing to its potential application in the detection of human carcinogenesis and in regulatory aspects. The assessment of GTIs during clinical development is a major liability. ${ }^{1-5)}$ Since GTIs are expected to be present in active pharmaceutical ingredients (APIs), a method for the sensitive and selective analysis of GTIs is required. The allowable limits of GTIs in new commercial drugs are regulated by government agencies such as the U.S. Food and Drug Administration (FDA) and the European Medicines Agency (EMA), ${ }^{6-9)}$ although the toxicological assessment of GTIs is not addressed sufficiently in the International Conference on Harmonisation $(\mathrm{ICH})$ guidelines. The present ICH Q3A and Q3B guidelines only describe the limits of conventional impurities and related substances, where typical levels are above $0.05 \%$. Therefore, the ICH has been discussing about GTIs as part of the ICH M7 guideline. The allowable limits of GTIs are decided by the U.S. FDA and the EMA depending on the duration and the daily dose of exposure during the clinical development. A toxicological threshold of $1.5 \mu \mathrm{g} / \mathrm{d}$ is suggested. Typically, the target detection limit (DL) in an API is $1 \mathrm{ppm}(1 \mu \mathrm{g} / \mathrm{g}$ API). Therefore, a sensitive and selective analytical method is required for their analysis.

4-Chloro-1-butanol, an alkylating agent, is one of the GTIs, which is generated when tetrahydrofuran (THF) and hydro-

\footnotetext{
The authors declare no conflict of interest.

${ }^{\dagger}$ Present address: Group II, Technology Department, Yoshitomi Plant, Mitsubishi Tanabe Pharma Factory Ltd.; 955 Koiwai, Yoshitomi-cho, Chikujogun, Fukuoka 871-8550, Japan.
}

chloric acid $(\mathrm{HCl})$ are used during the synthesis of APIs. The interaction of THF with $\mathrm{HCl}$ leads to the generation of 4-chloro-1-butanol (Fig. 1). THF and $\mathrm{HCl}$ are frequently used in the synthesis of APIs. Hence, it is necessary to confirm the fate of 4-chloro-1-butanol, to develop the process control for APIs and ensure their quality. The quantitation limit (QL), as per the analytical method, should be at low ppm levels, considering the protocols (the daily dose and the duration of exposure) of the clinical studies.

As part of the analytical method, conventional analytical instrumentations such as the HPLC with UV detection (for nonvolatile GTIs) or the GC with flame ionization detector (FID) detection (for volatile small molecules), should be employed

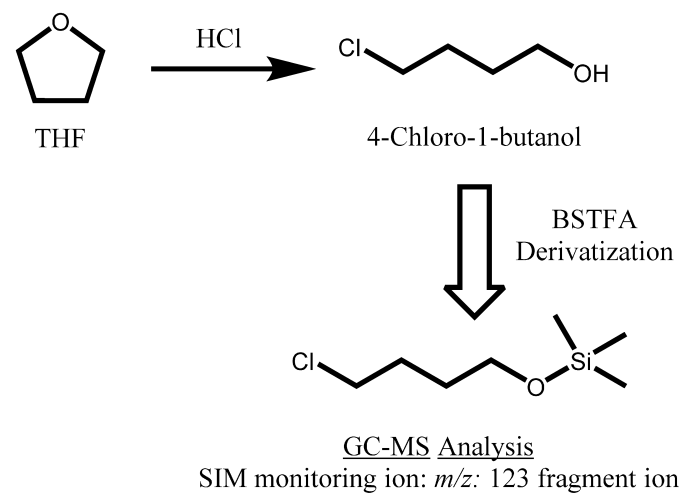

Fig. 1. Generation and Analysis of 4-Chloro-1-butanol

Interaction of THF with $\mathrm{HCl}$ leads to the generation of 4-chloro-1-butanol. $\mathrm{m} / \mathrm{z}$ : 123 fragment ion of the derivatized compound was monitored in the selected ion monitoring (SIM) mode by GC-MS. 
for the standard first attempt of GTI analysis. In general, for the determination of non-volatile small molecule GTIs, which have no UV chromophore, chemical derivatization is used to detect them using a UV detector and to retain them on the reversed-phase stationary phases such as an octadecylsilyl (ODS) silica gel, in the LC-UV method. On the other hand, in the GC-FID method, chemical derivatization is also employed to enhance the volality. However, for trace level detection, currently, either LC-MS or GC-MS is employed. ${ }^{10-12)}$ For the determination of 4-chloro-1-butanol in the APIs, the use of the GC-MS method ${ }^{12)}$ and the UV/VIS method, which uses Reichardt's dye (detection at $624 \mathrm{~nm})^{13)}$ has been reported. In this study, a sensitive GC-MS method was developed and validated. Silylation was employed to enhance the volatility of the non-volatile 4-chloro-1-butanol. Further, 3-chloro-1-butanol was used as an internal standard (IS) to ensure the accuracy and the precision of the method. To the best of our knowledge, there is no paper concerning a GC-MS with an IS method for the determination of small-molecule GTIs. For quality control tests in an API manufacturing plant, it is important to establish a test method that preferably employs conventional apparatus. It is advantageous to develop a flexible and versatile analytical method for routine analysis in quality control laboratories. For this reason, the authors chose non-labeled 3-chloro-1-butanol, which has a structure closely related to that of 4-chloro-1-butanol, as the IS even though isotope-labeled compounds are generally used as an IS. The multipurpose IS method using a non-labeled compound can applied to the GCFID method depending on the target DL. This approach was applied to the determination of 4-chloro-1-butanol in the API, which is under clinical trials.
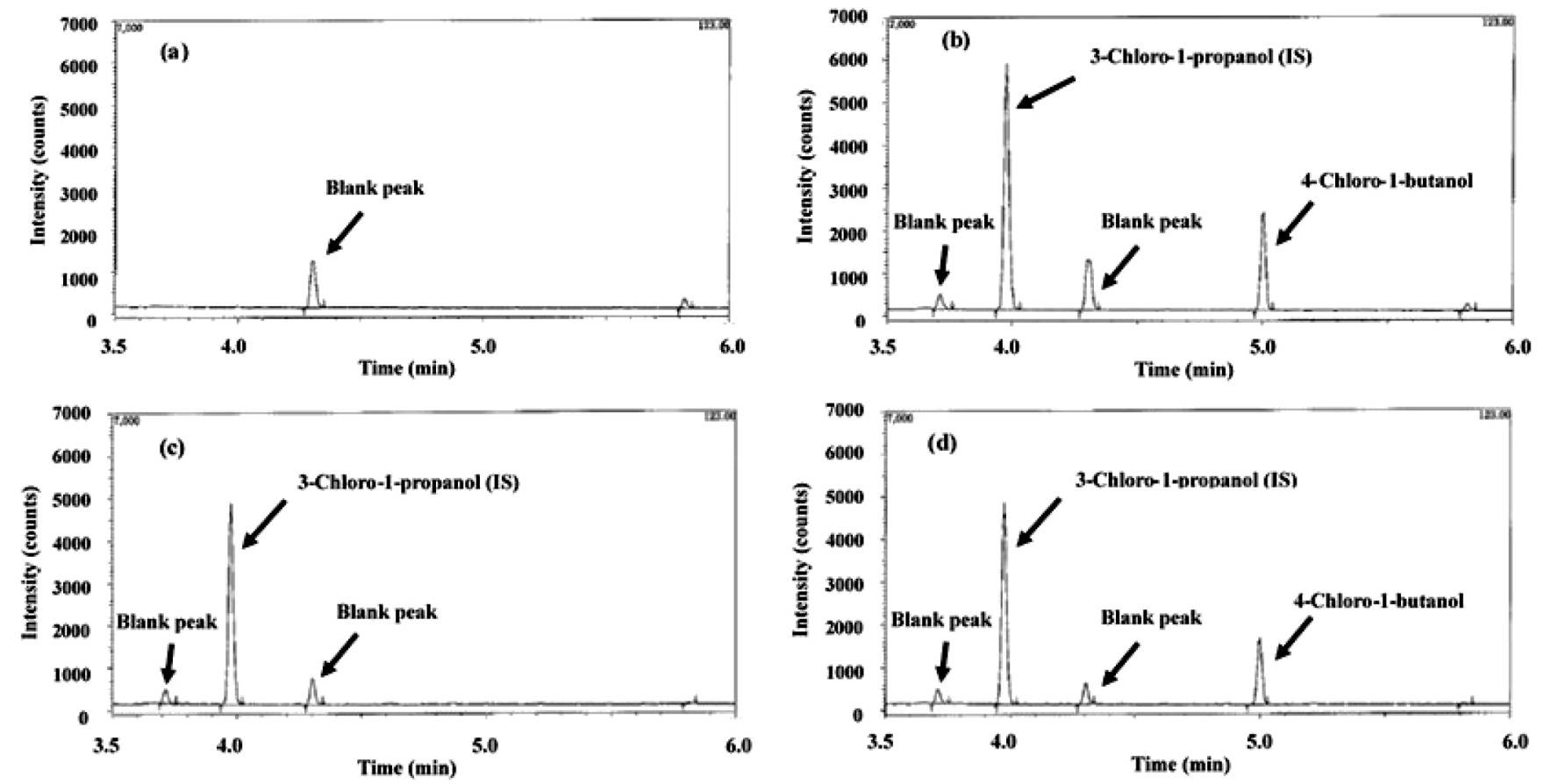

Chemicals and Reagents 4-Chloro-1-butanol (>97\% purity), 3-chloro-1-propanol ( $>97 \%$ purity) and $\mathrm{N}, \mathrm{O}$-bis(trimethylsilyl)trifluoroacetamide (BSTFA) ( $>95 \%$ purity), were purchased from Tokyo Chemical Industry (Tokyo, Japan). Ethyl acetate of the HPLC grade was purchased from Wako Pure Chemical Industries, Ltd. (Osaka, Japan). Helium gas ( $>99.999 \%$ purity) was purchased from Taiyo Nippon Sanso (Tokyo, Japan). Compound A (molecular weight: about 400), containing no functional groups such as a hydroxyl group or an amine, was manufactured by the Mistubishi Tanabe Pharma Corporation (Osaka, Japan).

Apparatus The GC-MS analysis was carried out using a GC-MS QP2010 Ultra instrument, with an autosampler AOC-20s, and an autoinjector AOC-20i (Shimadzu, Kyoto, Japan). Separation was performed using a DB-5MS capillary column $(0.25 \mathrm{~mm}$ i.d. $\times 30 \mathrm{~m}, 0.25 \mu \mathrm{m}$ film thickness, Agilent Technologies, Santa Clara, CA, U.S.A.). Helium was used as the carrier gas at a constant linear velocity of $30 \mathrm{~cm} / \mathrm{s}$. The oven temperature was initially maintained at $60^{\circ} \mathrm{C}$, and then programmed to rise at the rate of $15^{\circ} \mathrm{C} / \mathrm{min}$ to $280^{\circ} \mathrm{C}$ and then maintained at $280^{\circ} \mathrm{C}$ for $1 \mathrm{~min}$. A $2 \mu \mathrm{L}$ aliquot of the sample or standard solution was injected in the splitless mode. Both the inlet temperature and interface temperature were maintained at $250^{\circ} \mathrm{C}$. The temperature of the ion source was set at $200^{\circ} \mathrm{C}$. Ionization was carried out in the electron impact ionization mode (EI, $70 \mathrm{eV}$ ) and $m / z$ : 123 fragment ion of the derivatized compound was monitored in the selected ion monitoring (SIM) mode (Fig. 1).

Derivatization Reaction The derivatization of 4-chloro1-butanol and the IS (3-chloro-1-propanol) using BSTFA was carried out by adding $20 \mu \mathrm{L}$ of BSTFA to $2.5 \mathrm{~mL}$ ethyl acetate, containing ca. $25 \mathrm{mg}$ Compound $\mathrm{A}$ and $1 \mathrm{~mL}$ of the IS solution

\section{Experimental}

Fig. 2. GC-MS Chromatograms for Specificity

(a) Blank solution (containing no IS, 4-chloro-1-butanol, and Compound A), (b) standard solution (containing IS and 4ppm of 4-chloro-1-butanol), (c) sample solution (containing IS and Compound A) and (d) sample solution spiked with 4-chloro-1-butanol (containing IS, 4 ppm of 4-chloro-1-butanol, and Compound A). 
$(10 \mathrm{ng} / \mathrm{mL})$. Next, the solution was diluted to $10 \mathrm{~mL}$ with ethyl acetate. Then, the solution was incubated at $50^{\circ} \mathrm{C}$ for $20 \mathrm{~min}$.

Preparation of Standard and Sample Solutions The 4-chloro-1-butanol solutions were prepared at approximately $0.08,0.2,0.4,0.8,2,4,20$, and $40 \mathrm{ppm}(\mu \mathrm{g} / \mathrm{g}$ API $)$ using ethyl acetate. One ppm corresponds to $2.5 \mathrm{ng} / \mathrm{mL}$ of the 4-chloro1-butanol solution. These solutions were used for linearity, DL, and QL validation. The sample solution was prepared by using approximately $25 \mathrm{mg}$ of Compound A. A blank solution (without Compound A) was prepared using the same procedure. The sample solutions, spiked with $0.4,4$, and $20 \mathrm{ppm}$ of 4-chloro-1-butanol, were prepared in a triplicate. The standard solution was prepared at approximately $4 \mathrm{ppm}$. These solutions were used to validate the specificity, accuracy, and precision of the method. These solutions include 3-chloro-1-propanol as IS. The concentration of the IS is about $4 \mathrm{ppm}$.

Method Validation The specificity was validated using the blank solution, the standard solution (4 ppm of 4-chloro1-butanol), the sample solution, and the sample solution spiked with $4 \mathrm{ppm}$ of 4-chloro-1-butanol. The linearity was validated using triplicate injections of the $0.08,0.2,0.4,0.8,2,4,20$, and $40 \mathrm{ppm}$ solutions. The QL was confirmed using the relative standard deviation (R.S.D.) of the peak area ratio obtained from six injections of the $0.08 \mathrm{ppm}$ and $0.2 \mathrm{ppm}$ solutions. The DL was estimated from the slope of the calibration curve and the standard deviation of the response of the solution. The accuracy (recovery test) was validated by the standard solution $(4 \mathrm{ppm})$ and the sample solution spiked with three different concentrations $(0.4,4,20 \mathrm{ppm})$. We tested the standard and sample solutions in triplicate. The repeatability of the spiked recovery (three repetitions at three concentrations) was carried out to validate the precision. Range was validated from the results on linearity, accuracy, and precision.

Quantification of 4-Chloro-1-butanol in API About $25 \mathrm{mg}$ of Compound A was weighed and dissolved in $2.5 \mathrm{~mL}$ of ethyl acetate. Twenty microliters of BSTFA and $1 \mathrm{~mL}$ of the IS solution $(10 \mathrm{ng} / \mathrm{mL})$ were added to the solvent and the solution was diluted to $10 \mathrm{~mL}$ with ethyl acetate. Then, the solution was incubated at $50^{\circ} \mathrm{C}$ for $20 \mathrm{~min}$.

\section{Results and Discussion}

Derivatization Reaction and Optimization of the GC
Conditions Ethyl acetate was selected as the reaction solvent, considering the solubility of the sample. The GC oven temperature was programed appropriately to separate the interferences, such as the API and the derivatization reagent, from the analyte. The splitless mode was selected to increase the amount of sample injected into the GC-MS. To develop an accurate and robust GC determination method, the IS method was employed. 3-Chloro-1-propanol, which has a hydroxyl group and retention times similar to the analyte, was selected as the IS compound. Incidentally, the interaction of THF with $\mathrm{HCl}$ leads to the ring-opening reaction and the generation of 4-chloro-1-butanol; 3-chloro-1-propanol is not generated in the reaction. Moreover, taking into consideration the API synthesis route, the probability of contamination of 3-chloro1-propanol is low. Infact, no peak of 3-chloro-1-propanol was detected in Compound A by GC-MS analysis. Under optimized conditions, baseline separation of the analyte and the IS was achieved within $6 \mathrm{~min}$ (see, Fig. 2) and the GC analysis was conducted within $16 \mathrm{~min}$. The optimized conditions are summarized in Table 1.

Method Validation Results The specificity of the analyte was confirmed by comparing the four chromatograms, as shown in Fig. 2. Except the blank solution, all other solutions contained 3-chloro-1-propanol (IS). In the chromatogram of

Table 1. GC-MS Conditions

\begin{tabular}{ll}
\hline \hline \multicolumn{1}{c}{ Parameter } & \multicolumn{1}{c}{ Value } \\
\hline Ionization mode & $\mathrm{EI}, 70 \mathrm{eV}$ \\
Ion source temperature & $\mathrm{A}$ constant temperature of about $200^{\circ} \mathrm{C}$ \\
Detector voltage & $0.9 \mathrm{kV}$ \\
Mass/Analyte & $\mathrm{m} / z: 123$ (SIM mode) \\
Column & DB-5MS $(0.25 \mathrm{~mm}$ i.d. $\times 30 \mathrm{~m}, 0.25 \mu \mathrm{m}$, \\
& Agilent Technologies $)$ \\
Column heater temperature & At a rate of $15^{\circ} \mathrm{C}$ per minute from 60 to \\
& $280^{\circ} \mathrm{C}$, where it is maintained for $1 \mathrm{~min}$ \\
Interface temperature & A constant temperature of about $250^{\circ} \mathrm{C}$ \\
Inlet mode & Splitless \\
Inlet temperature & A constant temperature of about $250^{\circ} \mathrm{C}$ \\
Carrier gas & Helium $($ Constant linear velocity: $30 \mathrm{~cm} / \mathrm{s})$ \\
Integration time & $3.5-6 \mathrm{~min}$ \\
Injection volume & $2 \mu \mathrm{L}$ \\
\hline
\end{tabular}

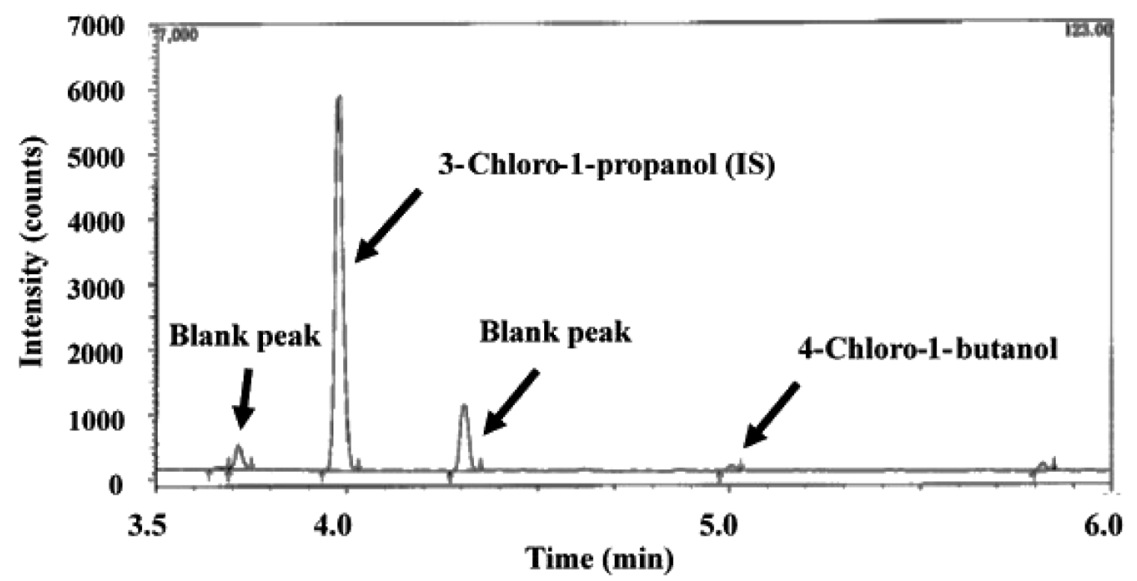

Fig. 3. Representative Chromatogram of 4-Chloro-1-butanol (0.08 ppm) for QL

The R.S.D. value of six injections of the $0.08 \mathrm{ppm}$ solution was $7.4 \%$. 
Table 2. Method Validation Results for Accuracy

\begin{tabular}{lccr}
\hline \hline \multirow{2}{*}{\multicolumn{1}{c}{ Accuracy }} & \multicolumn{3}{c}{ Recovery (\%) } \\
\cline { 2 - 4 } & $n=1$ & $n=2$ & $n=3$ \\
\hline Spiked with 0.4ppm & 107.0 & 90.5 & 101.5 \\
Spiked with 4 ppm & 108.7 & 108.6 & 96.9 \\
Spiked with 20 ppm & 106.4 & 106.8 & 105.5 \\
Average (\%) & & 103.5 & \\
Standard deviation (\%) & & 6.24 \\
Relative standard deviation (\%) & & 6.0 \\
\hline
\end{tabular}

the blank solution, no peak was observed around the retention times of 4-chloro-1-butanol ( $c a .5 \mathrm{~min}$ ) and the IS ( $c a .4 \mathrm{~min})$ derivatized with BSTFA (Fig. 2a). In the chromatogram of the sample solution, there is no peak around $5 \mathrm{~min}$, where the derivatized 4-chloro-1-butanol compound is detected. The linearity, ranging from 0.08 to $40 \mathrm{ppm}$, was obtained with an $R^{2}$ value of 0.9999 . The DL was estimated from the slope of the calibration curve and the standard deviation of the response of the solution $(3 \sigma)$. The DL was $0.05 \mathrm{ppm}$, which corresponds to $0.13 \mathrm{ng} / \mathrm{mL}$ of the 4 -chloro-1-butanol solution. The R.S.D. values of the six injections of the $0.08 \mathrm{ppm}$ and $0.2 \mathrm{ppm}$ solutions were $7.4 \%$ and $6.7 \%$, respectively. Incidentally, the R.S.D. value of the peak area of 4-chloro-1-butanol (calculated without an IS) was $10.4 \%$ and $11.4 \%$, respectively. This result indicates that the developed method has the advantage of precision. The QL was $0.08 \mathrm{ppm}$, which corresponds to $0.20 \mathrm{ng} /$ $\mathrm{mL}$ of the 4-chloro-1-butanol solution. The data obtained in the accuracy and precision studies are summarized in Table 2. The accuracy (recovery test) was in the range of $90.5 \%$ to $108.7 \%$ between $0.4 \mathrm{ppm}$ and $20 \mathrm{ppm}$. Moreover, the R.S.D. in the repeatability of the spiked recovery test was $6.0 \%$. A representative chromatogram at the quantitation limit is shown in Fig. 3. The results on linearity, accuracy, and precision support the suitability of this method in detecting trace amounts of 4-chloro-1-butanol over the range $0.4-20 \mathrm{ppm}$. Thus, this GC-MS method, using BSTFA derivatization, allows the trace analysis of 4-chloro-1-butanol. In addition, since BSTFA is generally employed as a derivatization reagent that can react not only with amine groups but also with hydroxyl groups, the developed method can be applied to the analysis of many other GTIs. However, it is necessary to justify the conditions such as the derivatization.

Quantification of 4-Chloro-1-butanol in APIs Since THF and $\mathrm{HCl}$ are used in the final synthesis process of Compound $\mathrm{A}$, there is a possibility that 4 -chloro-1-butanol remains in Compound A. The residual amount of 4-chloro-1-butanol in Compound A was confirmed by the developed GC-MS method. The resultant residual amount of 4-chloro-1-butanol in Compound A was below the QL of $0.08 \mathrm{ppm}$.

\section{Conclusion}

A GC-MS method for the trace level analysis of 4-chloro1-butanol in the API was developed. The DL and the QL were $0.05 \mathrm{ppm}$ and $0.08 \mathrm{ppm}$, respectively. The results of the validation study proved that the method was suitable for the analysis of trace amounts of GTI.

Acknowledgments The authors gratefully acknowledge Tunenobu Katsumata, Analytical Research Laboratories, CMC Division, and Mitsubishi Tanabe Pharma Corporation, for their technical assistance.

\section{References}

1) Callis C. M., Bercu J. P., DeVries K. M., Dow L. K., Robbins D. K., Varie D. L., Org. Process Res. Dev., 14, 986-992 (2010).

2) Kirkland D., Snodin D., Int. J. Pharm. Med., 18, 197-207 (2004).

3) Robinson D. I., Org. Process Res. Dev., 14, 946-959 (2010).

4) Yuabova Z. Y., Holschlag D. R., Rodriguez S. A., Qin C., Papov V. V., Qiu F., McCaffrey J. F., Norwood D. L., J. Liquid Chromatogr. Relat. Technol., 31, 2318-2330 (2008).

5) Teasdale A., "Genotoxic Impurities: Strategies for Identification and Control," John Wiley and Sons, Hoboken, NJ, 2010.

6) U.S. FDA, "Guidance for Industry: Genotoxic and Carcinogenic Impurities in Drug Substances and Products, Recommended Approaches." Draft, December 2008.

7) EMA, "Guideline on the limits of genotoxic impurities," CPMP/ SWP/5199/02, EMEA/CHMP/QWP/251344/2006, 28 June, 2006.

8) EMA, "Questions and Answers on the Guideline on the limits of genotoxic impurities," EMEA/CHMP/SWP/431994/2007, 23 September, 2010.

9) Müller L., Mauthe R. J., Riley C. M., Andino M. M., De Antonis D., Beels C., DeGeorge J., De Knaep A. G. M., Ellison D., Fagerland J. A., Frank R., Fritschel B., Galloway S., Harpur E., Humfrey C. D. N., Jacks A. S., Jagota N., Mackinnon J., Mohan G., Ness D. K., O’Donovan M. R., Smith M. D., Vudathala G., Yotti L., Regul. Toxicol. Pharmacol., 44, 198-211 (2006).

10) Elder D. P., Lipczynski A. M., Teasdale A., J. Pharm. Biomed. Anal., 48, 497-507 (2008).

11) Liu D. Q., Sun M., Kord A. S., J. Pharm. Biomed. Anal., 51, 999 1014 (2010).

12) David F., Jacq K., Sandra P., Baker A., Klee M. S., Anal. Bioanal. Chem., 396, 1291-1300 (2010).

13) Corrigan D. K., Whitcombe M. J., McCrossen S., Piletsky S., J. Pharm. Pharmacol., 61, 533-537 (2009). 